\title{
Labeling a kind of Cubic Graphs by Subgraph Embedding Method
}

\author{
Yujie. Bai
}

School of Mathematics and Information Science, Henan Polytechnic University, Henan, China E-mail: 15039113163@163.com

\section{Shufei. Wu}

School of Mathematics and Information Science, Henan Polytechnic University, Henan, China

E-mail: shufeiwu@ hotmail.com

Received: 01 January 2021; Accepted: 15 January 2021; Published: 08 February 2021

\begin{abstract}
Based on a problem raised by Gao et. al. (Bull. Malays. Math. Sci. Soc., 41 (2018) 443-453.), we construct a family of cubic graphs which are double-edge blow-up of ladder graphs. We determine the full friendly index sets of these cubic graphs by embedding labeling graph method. At the same time, the corresponding labeling graphs are provided.
\end{abstract}

Index Terms: Vertex labeling, Friendly labeling, Double-edge blow-up, $P_{2}$-embedding, $C_{4}$-embedding.

\section{Introduction}

Let $G$ be a simple graph with vertex set $V(G)$ and edge set $E(G)$. A vertex $0-1$ labeling $f$ induces an edge $0-1$ labeling $f^{+}$, given by $f^{+}(u v) \equiv f(u)+f(v) \quad(\bmod 2)$ for each $u v \in E(G)$. For $i \in\{0,1\}$, a vertex (resp. an edge) is called an $i$-vertex (resp. $i$-edge if it is labeled by $i$. An edge is also called an $(i, j)$-edge if it is incident with both an $i$-vertex and a $j$-vertex.

Define $v_{f}(i)=|\{u \in V(G): f(u)=i\}|$ and $e_{f}(i)=\left|\left\{e \in E(G): f^{+}(e)=i\right\}\right|$. A vertex labeling $f$ is said to be friendly if $\left|v_{f}(1)-v_{f}(0)\right| \leq 1$. For a friendly labeling $f$ of $G, I_{f}(G)=e_{f}(1)-e_{f}(0)$ is called the friendly index of $G$; and if $e_{f}(1)-e_{f}(0)=a$ then the labeling graph $G$ is denoted by $G_{f}(a)$. The full friendly index set, introduced by Shiu and Kwong[16], is the set $F F I(G)=\left\{e_{f}(1)-e_{f}(0): f\right.$ is a friendly labeling of $\left.G\right\}$. When the context is clear, we will drop the subscript $f$. In our figures of labeling graphs, the black points are labeled by 1 and the white points are labeled by 0 .

Graph labeling theory has numerous applications in the field of parallel computing, very-large-scale integration design, etc. Generally speaking, it is difficult to obtain the full friendly index sets of an arbitrary graph. Researchers usually focus their study on some specific graphs. For example, the full friendly index sets of $P_{2} \times P_{n}$ was determined by Shiu and Kwong[16]. The full friendly index sets of $C_{m} \times C_{n}$ was determined by Shiu and Ling [13]. In [12], Shiu and Lee determined the full friendly index sets of twisted cylinders. In [14] and [15], Sinha and Kaur investigated the full friendly index sets complete graphs, cycles, fans, double fans, wheels, double stars, $P_{3} \times P_{n}$, and the tensor product of $P_{2}$ and $P_{n}$. Law [8] investigated the full friendly index sets of spiders and disproved a conjecture by Salehi and Lee [11] that the friendly index sets of a tree forms an arithmetic progression. Sun, Gao, and Lee [11] determined the full friendly index sets for the twisted product of Möbius ladders and they also determined the full friendly index sets of $P_{m} \times P_{n}$ [4]. More results about full friendly index sets can be found in [10, 17-19].

Recently, Gao et. al. [2,3] introduced the embedding labeling graph method and obtained the full friendly index sets of some kinds of cubic graphs. They asked in [2] for other types of cubic graphs, whose full friendly index sets can be obtained by embedding labeling graph method? In this paper, we obtain the full friendly index sets of a family of cubic graphs which are double-edge blow-up of $P_{2} \times P_{n}, n \geq 2$. 
Definition 1. ([3]) Let $G$ and $H$ be two graphs such that $u$ and $v$ are two particular vertices of $H$. An edge $x y$ of $G$ is blown-up by $H$ at $u$ and if $x y$ is replaced by $H$ by identifying $x$ and $u$, and $y$ and $v$ respectively.

Let $P_{2} \times P_{n}(n \geq 2)$ be the ladder graph with vertex set $V=\left\{u_{i}, v_{i}: 1 \leq i \leq n\right\}$ and edge set $E=\left\{u_{i} u_{i+1}, v_{i} v_{i+1}: 1 \leq i \leq n-1\right\} \cup\left\{u_{i} v_{i}: 1 \leq i \leq n\right\}$, such that $|V|=2 n$ and $|E|=3 n-2$.

Let $K_{3,3}^{-}$be the bipartite graph obtained by deleting an edge from $K_{3,3}$, suppose the two 2-degree vertices arrived are $u$ and $v$.

Definition 2. The double-edge blow-up graph of $P_{2} \times P_{n}$, denoted by $H_{n-2}$, is obtained by blowing up the edges $u_{1} v_{1}$ and $u_{n} v_{n}$ of $P_{2} \times P_{n}$ by two disjoint copies of $K_{3,3}^{-}$at its $u, v$ vertices respectively.

For easy of notations, we let $m=n-2$. By definition 2, we know that $H_{m}$ is a cubic graph of order $2 m+12$ and size $3 m+18$. See $H_{1}$ in Figure 1 as an example.

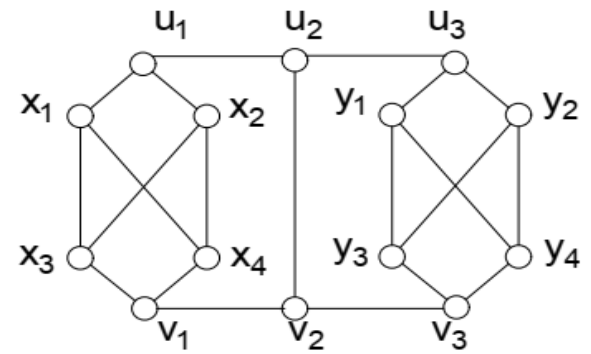

Fig.1. Graph $H_{1}$.

We end this section with some notations. As usual, $[k]$ stands for $\{1,2, \cdots, k\}$. For a graph $G$ and two vertex subsets $X, Y$ of $V(G)$, we use $e(X)$ to denote the number of edges with both ends in $X$. We use $e(X, Y)$ to denote the number of edges with one end in $X$ and the other in $Y$.

\section{Some Basic Properties}

The following lemma is concerned with the number of 1-edges in a labeling cycle. It says each cycle meets each edge cut with an even number of edges. We provide a proof by using the graph partitioning theory, which is closed related to graph labelings. For more problems and results on graph partitions, we refer the reader to [5, 6].

Lemma 3 ([17]). Let $f$ be a labeling of a graph $G$ that contains a cycle $C$ as its subgraph. If $C$ contains a 1 -edge, then the number of 1 -edges in $C$ is a positive even number.

Proof: For $i=1,2$, let $V_{i}$ be the set of vertices with label $i$. Then

$$
e(1)=e\left(V_{0}, V_{1}\right)=\sum_{v \in V_{0}} d(v)-2 e\left(V_{0}\right)=2\left|V_{0}\right|-2 e\left(V_{0}\right)
$$

which is an even integer.

Staring with a friendly labeling of a graph, to get all possible friendly indices, an effective way is to exchanging vertices labels. The following lemma shows how the friendly index changes by changing vertices labels.

Lemma 4. Let $f_{1}$ be a friendly labeling of a cubic graph and $f_{2}$ is obtained from $f_{1}$ by exchanging two distinct vertices labels, then

$$
\left(e_{f_{1}}(1)-e_{f_{1}}(0)\right)-\left(e_{f_{2}}(1)-e_{f_{2}}(0)\right) \equiv 0 \quad(\bmod 4) .
$$


Proof: Let $V_{1}$ (resp., $V_{1}^{\prime}$ ) be the set of vertices labelled 1 under $f_{1}$ (resp., $f_{2}$ ), then $\left|V_{1}\right|=\left|V_{1}^{\prime}\right|$. By considering the sum of degrees of vertices in $V_{1}$ and $V_{1}^{\prime}$, we obtain

$$
\sum_{v \in V_{1}} d(v)=3\left|V_{1}\right|=2 e\left(V_{1}\right)+e_{f_{1}}(1)
$$

and

$$
\sum_{v \in V_{1}^{\prime}} d(v)=3\left|V_{1}^{\prime}\right|=2 e\left(V_{1}^{\prime}\right)+e_{f_{2}}(1)
$$

Since $e_{f_{1}}(1)-e_{f_{1}}(0)=2 e_{f_{1}}(1)-|E|$, we have

$$
\begin{aligned}
& \left(e_{f_{1}}(1)-e_{f_{1}}(0)\right)-\left(e_{f_{2}}(1)-e_{f_{2}}(0)\right) \\
& =2\left(e_{f_{1}}(1)-e_{f_{2}}(1)\right) \\
& =2\left[\left(3\left|V_{1}\right|-2 e\left(V_{1}\right)\right)-\left(3\left|V_{1}^{\prime}\right|-2 e\left(V_{1}^{\prime}\right)\right)\right] \\
& =4\left(e\left(V_{1}^{\prime}\right)-e\left(V_{1}\right)\right) \\
& =0(\bmod 4) .
\end{aligned}
$$

Let $H_{m}(a)$ be the friendly labeling graph $H_{m}$ with $e(1)-e(0)=a$. We record the labels of a path-subgraph $u_{i} v_{i}$ in a labeled $H_{m}$ by a vector $\left[\begin{array}{c}f\left(u_{i}\right) \\ f\left(v_{i}\right)\end{array}\right]$; and record the labels of a cycle-subgraph $u_{i} u_{i+1} v_{i+1} v_{i}$ by a matrix $\left[\begin{array}{lll}f\left(u_{i}\right) & f\left(u_{i+1}\right) \\ f\left(v_{i}\right) & f\left(v_{i+1}\right)\end{array}\right]$. Now we introduce the embedding method.

Definition 5. A $P_{2}$-embedding onto $H_{m}(a)$ at edges $u_{i} u_{i+1}$ and $v_{i} v_{i+1}$ is obtained by replacing $u_{i} u_{i+1}$ and $v_{i} v_{i+1}$ by two length 2 paths $u_{i} a_{1} u_{i+1}$ and $v_{i} a_{2} v_{i+1}$ respectively and jointing vertices $a_{1}$ and $a_{2}$. A $P_{2}$-embedding is denoted by

$$
\left[\begin{array}{l}
f\left(a_{1}\right) \\
f\left(a_{2}\right)
\end{array}\right]+\left[\begin{array}{ll}
f\left(u_{i}\right) & f\left(u_{i+1}\right) \\
f\left(v_{i}\right) & f\left(v_{i+1}\right)
\end{array}\right] .
$$

For example, in $H_{0}(-14)$, embed $\left[\begin{array}{l}1 \\ 0\end{array}\right]+\left[\begin{array}{ll}1 & 0 \\ 1 & 0\end{array}\right]$ obtain $H_{1}(-15)$, see Figures 2 and 4.

Definition 6. A $C_{4}$-embedding onto $H_{m}(a)$ at edges $u_{i} u_{i+1}$ and $v_{i} v_{i+1}$ is obtained by replacing $u_{i} u_{i+1}$ and $v_{i} v_{i+1}$ by two length 3 paths $u_{i} b_{1} b_{2} u_{i+1}$ and $v_{i} b_{3} b_{4} v_{i+1}$ respectively. Then jointing vertices $b_{1}, b_{3}$ and vertices $b_{2}, b_{4}$ respectively. A $C_{4}$-embedding is denoted by

$$
\left[\begin{array}{ll}
f\left(b_{1}\right) & f\left(b_{2}\right) \\
f\left(b_{3}\right) & f\left(b_{4}\right)
\end{array}\right]+\left[\begin{array}{ll}
f\left(u_{i}\right) & f\left(u_{i+1}\right) \\
f\left(v_{i}\right) & f\left(v_{i+1}\right)
\end{array}\right] .
$$

For example, in $H_{0}(-14)$ embed $\left[\begin{array}{ll}1 & 0 \\ 1 & 0\end{array}\right]+\left[\begin{array}{ll}1 & 0 \\ 1 & 0\end{array}\right]$ we obtain $H_{2}(-20)$, see Figures 2 and 3.

Clearly, a $P_{2}$-embedding (resp., $C_{4}$-embedding) will adds three (resp., six) extra edges to a graph.

\section{The Full Friendly index sets of $H_{m}$}

In this section, we obtain the full friendly index sets of $H_{m}$ by a sequence of embedding process. Firstly, we show 
the minimum value and maximum value of $e(1)$ among all friendly labelings.

Lemma 7. For any friendly labeling fof $H_{m}$, we have

(1) $2 \leq e(1) \leq 3 m+18$ if $m \geq 0$ is even;

(2) $3 \leq e(1) \leq 3 m+18$ if $m>0$ is odd.

Proof: Let $x_{1}, x_{2}, x_{3}, x_{4}$ and $y_{1}, y_{2}, y_{3}, y_{4}$ be the 3-degree vertices of the two $K_{3,3}^{-}$-subgraphs in $H_{m}$ respectively (see Figure 1 for the case $m=1$ ).

Since $H_{m}$ is connected, we have $e(1) \geq 1$. Note that each edge belongs to some cycle of $H_{m}$, by Lemma 3 , we know that $e(1) \geq 2$.

When $m$ is even, since $\left|V\left(H_{m}\right)\right|=2(m+6)$ and the labeling $f$ of $H_{m}$ is friendly, we know $v(1)=v(0)=m+6$. To construct the labeling with $e(1)=2$, we label $x_{1}, x_{2}, x_{3}, x_{4}$ and $u_{i}, v_{i},\left(1 \leq i \leq \frac{m+2}{2}\right)$ by 1 and the remainders by 0 . The maximum value $3 m+18$ can be attained by labeling $x_{1}, x_{2}, y_{3}, y_{4}, u_{2}, u_{4}, \ldots, u_{m+2}, v_{1}, v_{3}, \ldots, v_{m+1}$ by 1 and the remainders by 0 .

When $m$ is odd, if $e(1)=2$ then by Lemma 3 each cycle contains one 1-edge must contain the other. Therefore, both of the two 1-edges will belong the same side (left or right) of edge $u_{\frac{m+3}{2}} v_{\frac{m+3}{2}}$, which implies the labeling $f$ of $H_{m}$ is not friendly. So $e(1) \geq 3$. The labeling with $e(1)=3$ can be get by labeling $x_{1}, x_{2}, x_{3}, x_{4}$ and $v_{i}\left(1 \leq i \leq \frac{m+1}{2}\right), u_{i}\left(1 \leq i \leq \frac{m+3}{2}\right)$ by 1 and the remainders by 0 . The maximum value of $e(1)$ is $3 m+18$ can be attained by labeling $x_{3}, x_{4}, y_{3}, y_{4}, u_{1}, u_{3}, \ldots, u_{m+2}, v_{2}, v_{4}, \ldots, v_{m+1}$ by 1 and the remainders by 0 . This completes the proof of Lemma 7.

When $m$ is even, first we show the $m=0$ and $m=2$ cases, then our result is proved by induction. When $m$ is odd, our proof is carried out by doing $P_{2}$-embedding based on the even cases. Note that we only do embedding of friendly labeled $P_{2}$ or $C_{4}$-subgraphs. Hence, if the labeling of $H_{m}$ is friendly, then so do $H_{m+1}$ and $H_{m+2}$.

Lemma 8. When $m \geq 0$ is even,

$$
F F I\left(H_{m}\right)=\left\{-3 m-18+4 i: 1 \leq i \leq \frac{3 m+18}{2}\right\}
$$

Proof: By Lemma 4 and Lemma 7, the left hand is a subset of the right. The labeling graphs in Figure 2 show that the equality holds when $m=0$.

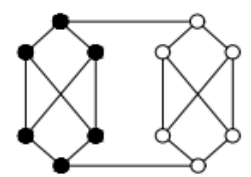

$e(1)-e(0)=-14$

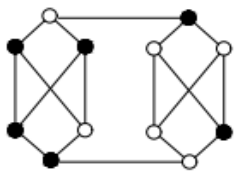

$\mathrm{e}(1)-\mathrm{e}(0)=6$

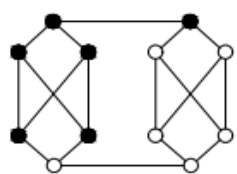

$e(1)-e(0)=-10$

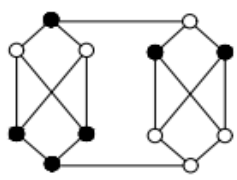

$e(1)-e(0)=10$

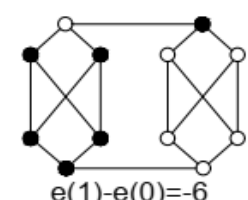

$e(1)-e(0)=-6$

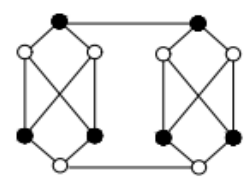

$e(1)-e(0)=14$
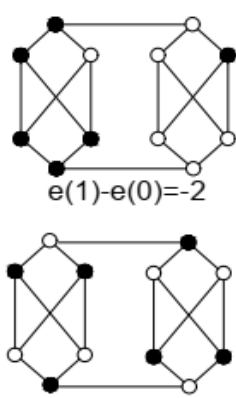

$e(1)-e(0)=18$

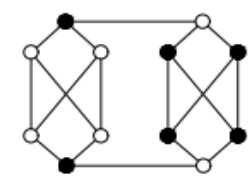

$e(1)-e(0)=2$

Fig.2. Labeling graphs $H_{0}$. 
(i) $m=2$

We show that there exist labeling graphs of $H_{2}(-24+4 i), 1 \leq i \leq 12$, by doing the following $C_{4}$-embeddings:

(1). In $H_{0}(-14)$ embed $\left[\begin{array}{ll}1 & 0 \\ 1 & 0\end{array}\right]+\left[\begin{array}{ll}1 & 0 \\ 1 & 0\end{array}\right]$, then $e(1)-e(0)$ decreased by 6 , we obtain $H_{2}(-20)$. In this labeling graph, there exists the labeling subgraph $\left[\begin{array}{ll}1 & 0 \\ 1 & 0\end{array}\right]$.

(2). In $H_{0}(-14), H_{0}(-2), H_{0}(2), H_{0}(10)$ embed

$$
\left[\begin{array}{ll}
1 & 1 \\
0 & 0
\end{array}\right]+\left[\begin{array}{ll}
1 & 0 \\
1 & 0
\end{array}\right]
$$

then $e(1)-e(0)$ decreased by 2 , we obtain $H_{2}(-16), H_{2}(-4), H_{2}(0), H_{2}(8)$. In these labeling graphs, there exists the labeling subgraph $\left[\begin{array}{ll}1 & 1 \\ 0 & 0\end{array}\right]$.

(3). In $H_{0}(-10), H_{0}(14)$, embed $\left[\begin{array}{ll}1 & 1 \\ 0 & 0\end{array}\right]+\left[\begin{array}{ll}1 & 1 \\ 0 & 0\end{array}\right]$, then $e(1)-e(0)$ decreased by 2 , we obtain $H_{2}(-12), H_{2}(12)$. In these labeling graphs, there exists the labeling subgraph $\left[\begin{array}{ll}1 & 1 \\ 0 & 0\end{array}\right]$.

(4). In $H_{0}(-6), H_{0}(6), H_{0}(18)$, embed $\left[\begin{array}{ll}1 & 1 \\ 0 & 0\end{array}\right]+\left[\begin{array}{ll}0 & 1 \\ 1 & 0\end{array}\right]$, then $e(1)-e(0)$ decreased by 2 , we obtain $H_{2}(-8)$, $H_{2}(4), H_{2}(16)$. In these labeling graphs, there exists the labeling subgraph $\left[\begin{array}{ll}1 & 1 \\ 0 & 0\end{array}\right]$.

(5). In $H_{0}(14)$, embed $\left[\begin{array}{ll}1 & 0 \\ 0 & 1\end{array}\right]+\left[\begin{array}{ll}1 & 1 \\ 0 & 0\end{array}\right]$, then $e(1)-e(0)$ increased by 6 , we obtain $H_{2}(20)$. In this labeling graph, there exists the labeling subgraph $\left[\begin{array}{ll}1 & 1 \\ 0 & 0\end{array}\right]$.

(6). In $H_{0}(18)$, embed $\left[\begin{array}{ll}1 & 0 \\ 0 & 1\end{array}\right]+\left[\begin{array}{ll}0 & 1 \\ 1 & 0\end{array}\right]$, then $e(1)-e(0)$ increased by 6 , we obtain $H_{2}(24)$. In this labeling graph, there exists the labeling subgraph $\left[\begin{array}{ll}0 & 1 \\ 1 & 0\end{array}\right]$.

The labeling graphs of $\mathrm{H}_{2}$ are shown in Figure 3.
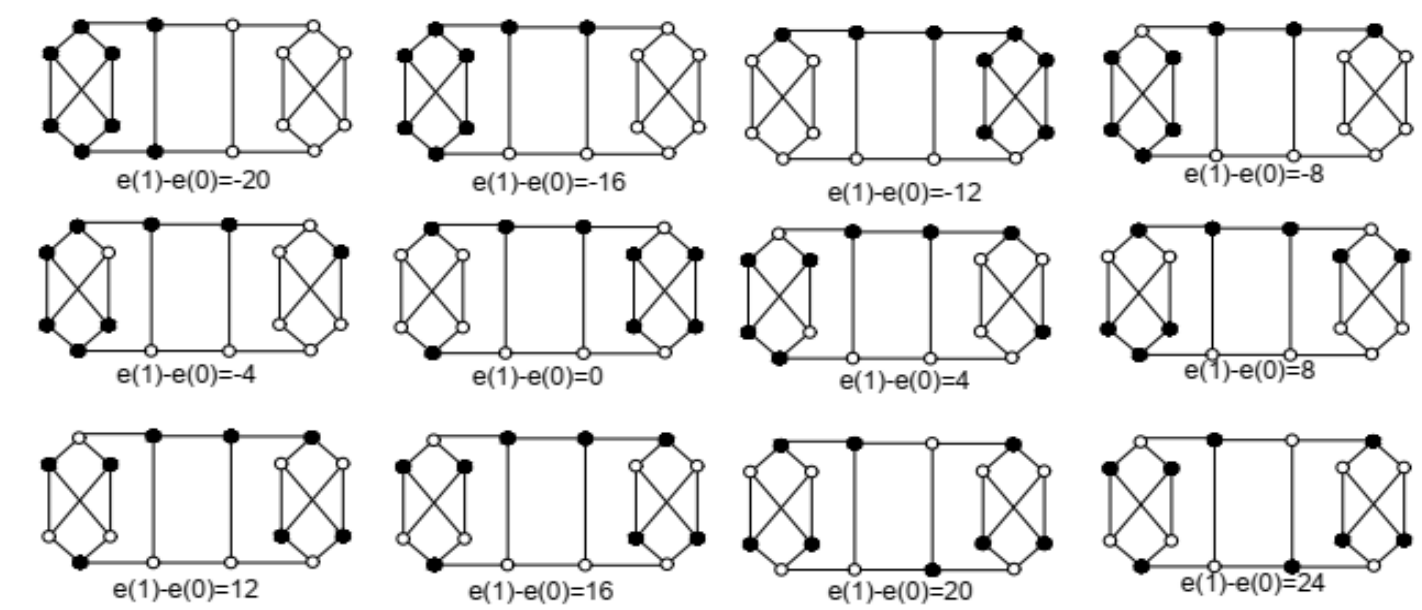

Fig.3. Labeling graphs $\mathrm{H}_{2}$. 
In conclusion, $F F I\left(H_{2}\right)=\{-24+4 i: 1 \leq i \leq 12\}$.

(ii) $m=4$

(1). In $H_{2}(-20)$, embed $\left[\begin{array}{ll}1 & 0 \\ 1 & 0\end{array}\right]+\left[\begin{array}{ll}1 & 0 \\ 1 & 0\end{array}\right]$ and $\left[\begin{array}{ll}1 & 1 \\ 0 & 0\end{array}\right]+\left[\begin{array}{ll}1 & 0 \\ 1 & 0\end{array}\right]$, then $e(1)-e(0)$ decreased by 6 and 2 respectively, we obtain $H_{4}(-26)$ and $H_{4}(-22)$. In these labeling graphs, there exists the labeling subgraphs $\left[\begin{array}{ll}1 & 0 \\ 1 & 0\end{array}\right]$ and $\left[\begin{array}{ll}1 & 1 \\ 0 & 0\end{array}\right]$ respectively.

(2). In $H_{2}(-24+4 i), 2 \leq i \leq 11$, there exists labeling subgraph $\left[\begin{array}{ll}1 & 1 \\ 0 & 0\end{array}\right]$. We do embedding $\left[\begin{array}{ll}1 & 1 \\ 0 & 0\end{array}\right]+\left[\begin{array}{ll}1 & 1 \\ 0 & 0\end{array}\right]$ then $e(1)-e(0)$ decrease by 2 , we obtain $H_{4}(-30+4 i), 3 \leq i \leq 12$. In these labeling graphs, there exists the labeling $\operatorname{subgraph}\left[\begin{array}{ll}1 & 1 \\ 0 & 0\end{array}\right]$.

(3). In $H_{2}(20)$, embed $\left[\begin{array}{ll}1 & 0 \\ 0 & 1\end{array}\right]+\left[\begin{array}{ll}1 & 1 \\ 0 & 0\end{array}\right]$, then $e(1)-e(0)$ increase by 6 , we obtain $H_{4}(26)$. In this labeling graph, there exists the labeling subgraph $\left[\begin{array}{ll}1 & 1 \\ 0 & 0\end{array}\right]$.

(4). In $H_{2}(24)$, embed $\left[\begin{array}{ll}1 & 1 \\ 0 & 0\end{array}\right]+\left[\begin{array}{ll}0 & 1 \\ 1 & 0\end{array}\right]$ and $\left[\begin{array}{ll}0 & 1 \\ 1 & 0\end{array}\right]+\left[\begin{array}{ll}0 & 1 \\ 1 & 0\end{array}\right]$, then $e(1)-e(0)$ decrease by 2 and $e(1)-e(0)$ increase by 6 respectively, we get $H_{4}(22)$ and $H_{4}(30)$. In thiese labeling graphs, there exists the labeling subgraphs $\left[\begin{array}{ll}1 & 1 \\ 0 & 0\end{array}\right]$ and $\left[\begin{array}{ll}0 & 1 \\ 1 & 0\end{array}\right]$.

Hence, $F F I\left(H_{4}\right)=\{-30+4 i: 1 \leq i \leq 15\}$.

(iii) $m \geq 6$

We assume that for even $k \geq 6, F F I\left(H_{k}\right)=\left\{-3 k-18+4 i: 1 \leq i \leq \frac{3 k+18}{2}\right\}$. We do embeddings as follow.

(1). In $H_{k}(-3 k-14)$, embed $\left[\begin{array}{ll}1 & 0 \\ 1 & 0\end{array}\right]+\left[\begin{array}{ll}1 & 0 \\ 1 & 0\end{array}\right]$ and $\left[\begin{array}{ll}1 & 1 \\ 0 & 0\end{array}\right]+\left[\begin{array}{ll}1 & 0 \\ 1 & 0\end{array}\right]$, then $e(1)-e(0)$ decreased by 6 and 2 respectively, we get $H_{k+2}(-3(k+2)-14)$ and $H_{k+2}(-3(k+2)-10)$. In these labeling graphs, there exists the labeling subgraphs $\left[\begin{array}{ll}1 & 0 \\ 1 & 0\end{array}\right]$ and $\left[\begin{array}{ll}1 & 1 \\ 0 & 0\end{array}\right]$ respectively.

(2). In $H_{k}(-3 k-18+4 i), 2 \leq i \leq \frac{3 k+16}{2}$, there exists labeling subgraph $\left[\begin{array}{ll}1 & 1 \\ 0 & 0\end{array}\right]$. We do embedding $\left[\begin{array}{ll}1 & 1 \\ 0 & 0\end{array}\right]+\left[\begin{array}{ll}1 & 1 \\ 0 & 0\end{array}\right]$, then $e(1)-e(0)$ decrease by 2 , we obtain $H_{k+2}(-3(k+2)-18+4 i), 3 \leq i \leq \frac{3(k+2)+12}{2}$. In these labeling graphs, there exists the labeling subgraph $\left[\begin{array}{ll}1 & 1 \\ 0 & 0\end{array}\right]$.

(3). In $H_{k}(3 k+14)$, embed $\left[\begin{array}{ll}1 & 0 \\ 0 & 1\end{array}\right]+\left[\begin{array}{ll}1 & 1 \\ 0 & 0\end{array}\right]$ then $e(1)-e(0)$ increased by 6 . Hence, we obtain $H_{k+2}(3(k+2)+14)$. In this labeling graph, there exists the labeling subgraph $\left[\begin{array}{ll}1 & 1 \\ 0 & 0\end{array}\right]$.

(4). In $H_{k}(3 k+18)$, embed $\left[\begin{array}{ll}1 & 1 \\ 0 & 0\end{array}\right]+\left[\begin{array}{ll}0 & 1 \\ 1 & 0\end{array}\right]$ and $\left[\begin{array}{ll}0 & 1 \\ 1 & 0\end{array}\right]+\left[\begin{array}{ll}0 & 1 \\ 1 & 0\end{array}\right]$, then $e(1)-e(0)$ decreased by 2 and increase $e(1)-e(0)$ by 6 respectively, we get $H_{k+2}(3(k+2)+10)$ and $H_{k+2}(3(k+2)+18)$. In these labeling graphs, 
there exists the labeling subgraphs $\left[\begin{array}{ll}0 & 1 \\ 1 & 0\end{array}\right]$.

By induction, we have $F F I\left(H_{k+2}\right)=\left\{-3(k+2)-18+4 i: 1 \leq i \leq \frac{3(k+2)+18}{2}\right\}$.

Lemma 9. When $m \geq 1$ is odd,

$$
F F I\left(H_{m}\right)=\left\{-3 m-16+4 i: 1 \leq i \leq \frac{3 m+17}{2}\right\}
$$

Proof: By Lemma 4 and Lemma 7, the left hand is a subset of the right. We prove the opposite direction by doing $P_{2}$ embeddings. We first show that the equality holds when $m=1,3$.

(i) $m=1$

We show that there exist labeling graphs of $H_{1}(-19+4 i), 1 \leq i \leq 10$, by doing the following $P_{2}$-embeddings:

(1). In $H_{0}(-14), H_{0}(-2), H_{0}(2), H_{0}(10)$ embed $\left[\begin{array}{l}1 \\ 0\end{array}\right]+\left[\begin{array}{ll}1 & 0 \\ 1 & 0\end{array}\right]$, then $e(1)-e(0)$ decreased by 1 , we obtain $H_{1}(-15), H_{1}(-3), H_{1}(1), H_{1}(9)$.

(2). In $H_{0}(-6), H_{0}(6), H_{0}(18)$, embed $\left[\begin{array}{l}1 \\ 0\end{array}\right]+\left[\begin{array}{ll}0 & 1 \\ 1 & 0\end{array}\right]$, then $e(1)-e(0)$ decreased by 1 , we obtain $H_{1}(-7)$, $H_{1}(5), H_{1}(17)$.

(3). In $H_{0}(-10), H_{0}(14)$, embed $\left[\begin{array}{l}1 \\ 0\end{array}\right]+\left[\begin{array}{ll}1 & 1 \\ 0 & 0\end{array}\right]$, then $e(1)-e(0)$ decreased by 1 , we obtain $H_{1}(-11), H_{1}(13)$.

(4). In $H_{0}(14)$, embed $\left[\begin{array}{l}0 \\ 1\end{array}\right]+\left[\begin{array}{ll}1 & 1 \\ 0 & 0\end{array}\right]$, then $e(1)-e(0)$ increased by 7 , we obtain $H_{1}(21)$.

The labeling graphs of $H_{1}$ are shown in Figure 4.

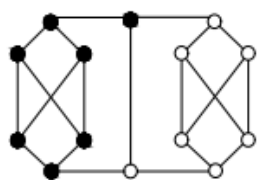

$e(1)-e(0)=-15$

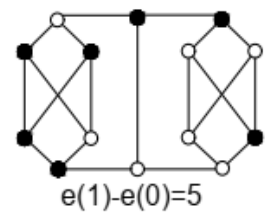

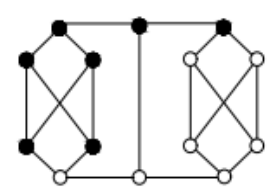

$e(1)-e(0)=-11$

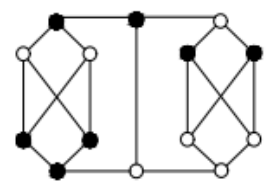

$e(1)-e(0)=9$
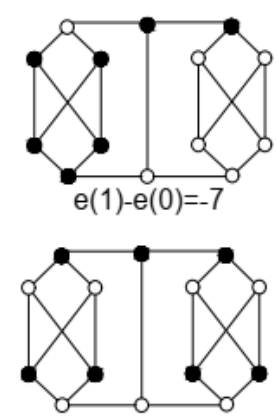

$e(1)-e(0)=13$

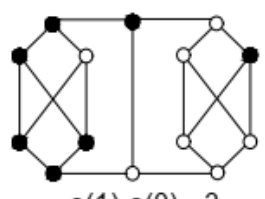

$e(1)-e(0)=-3$

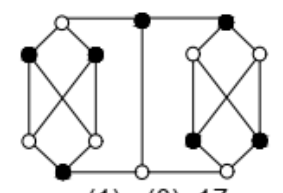

$e(1)-e(0)=17$

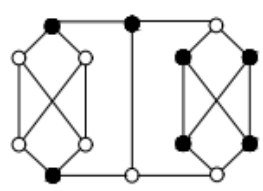

$e(1)-e(0)=1$

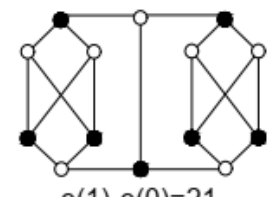

$e(1)-e(0)=21$

Fig.4. Labeling graphs $H_{1}$.

In summary, $F F I\left(H_{1}\right)=\{-19+4 i: 1 \leq i \leq 10\}$.

(ii) $m=3$

(1). In $H_{2}(-20)$, embed $\left[\begin{array}{l}1 \\ 0\end{array}\right]+\left[\begin{array}{ll}1 & 0 \\ 1 & 0\end{array}\right]$, then $e(1)-e(0)$ decreased by 1 , we obtain $H_{3}(-21)$.

(2). In $H_{2}(-24+4 i), 2 \leq i \leq 11$, there exists labeling subgraph $\left[\begin{array}{ll}1 & 1 \\ 0 & 0\end{array}\right]$. We do embedding $\left[\begin{array}{l}1 \\ 0\end{array}\right]+\left[\begin{array}{ll}1 & 1 \\ 0 & 0\end{array}\right]$, then $e(1)-e(0)$ decreased by 1 , we obtain $H_{3}(-25+4 i), 2 \leq i \leq 11$.

(3). In $H_{2}(24)$, embed $\left[\begin{array}{l}1 \\ 0\end{array}\right]+\left[\begin{array}{ll}0 & 1 \\ 1 & 0\end{array}\right]$, then $e(1)-e(0)$ decreased by 1 , we obtain $H_{3}(23)$. 
(4). In $H_{2}(20)$, embed $\left[\begin{array}{l}0 \\ 1\end{array}\right]+\left[\begin{array}{ll}1 & 1 \\ 0 & 0\end{array}\right]$, then $e(1)-e(0)$ increased by 7 , we obtain $H_{3}(27)$.

The labeling graphs of $H_{3}$ are shown in Figure 5.
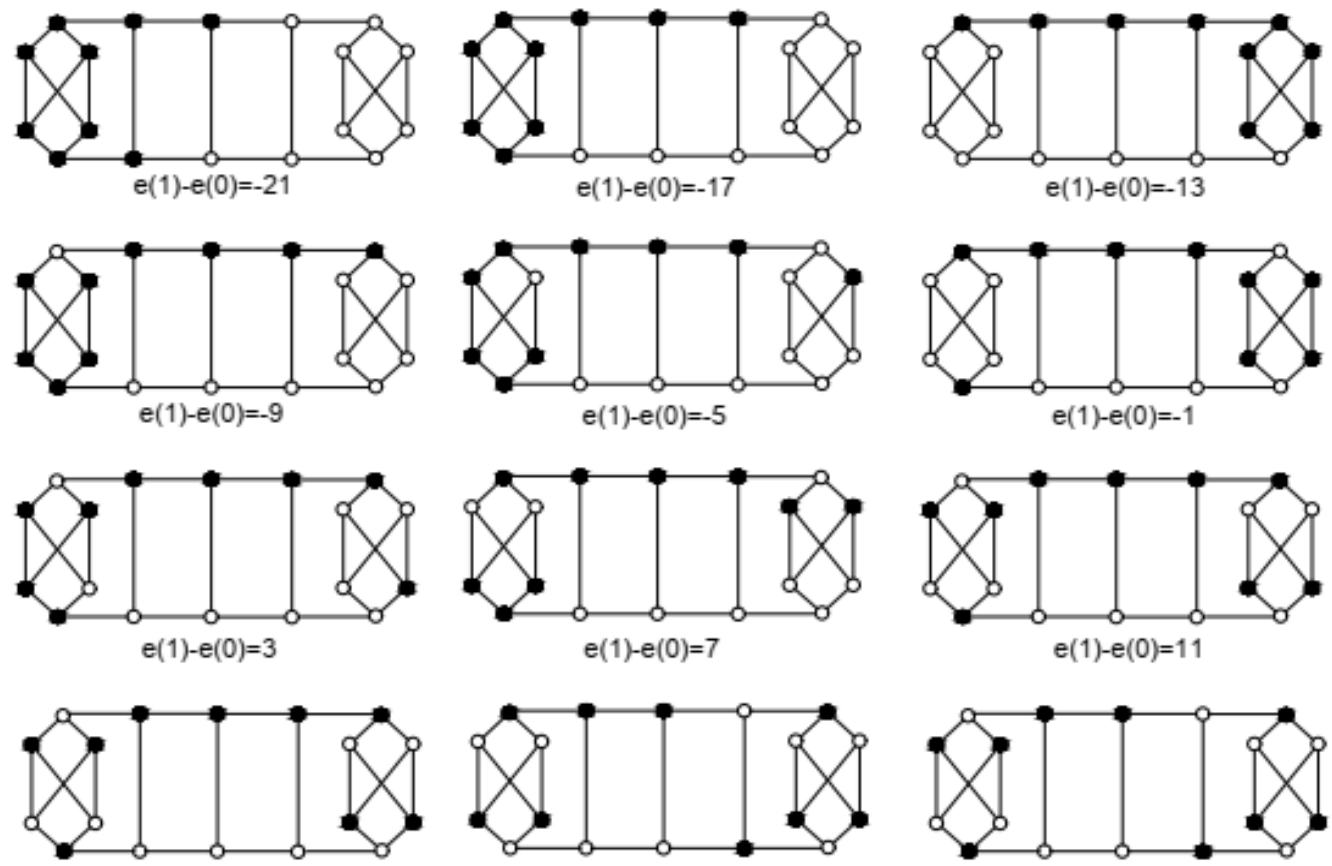

$e(1)-e(0)=19$
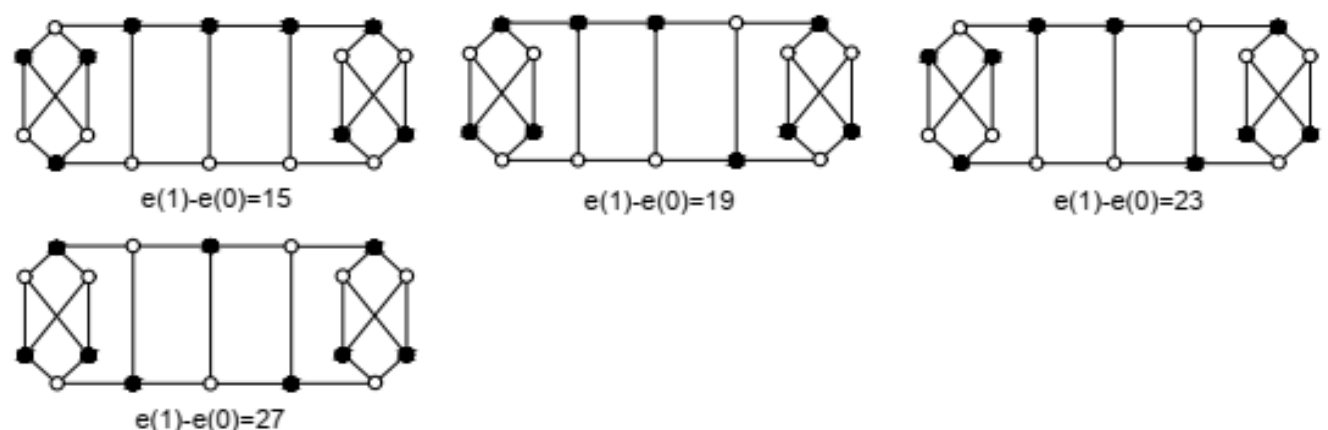

Fig.5. Labeling graphs $H_{3}$.

Hence, $F F I\left(H_{3}\right)=\{-24+4 i: 1 \leq i \leq 13\}$.

(iii) $m \geq 5$

We assume that for even $k \geq 4$ so $k+1$ is odd and $k+1 \geq 5$, $F F I\left(H_{k}\right)=\left\{-3 k-18+4 i: 1 \leq i \leq \frac{3 k+18}{2}\right\}$. We do embeddings as follow.

(1). In $H_{k}(-3 k-14)$, embed $\left[\begin{array}{l}1 \\ 0\end{array}\right]+\left[\begin{array}{ll}1 & 0 \\ 1 & 0\end{array}\right]$, then $e(1)-e(0)$ decreased by 1 , we obtain $H_{k+1}(-3(k+1)-12)$.

(2). In $H_{k}(-3 k-18+4 i), 2 \leq i \leq \frac{3 k+16}{2}$, there exists labeling subgraph $\left[\begin{array}{ll}1 & 1 \\ 0 & 0\end{array}\right]$. We do embedding $\left[\begin{array}{l}1 \\ 0\end{array}\right]+\left[\begin{array}{ll}1 & 1 \\ 0 & 0\end{array}\right]$, then $e(1)-e(0)$ decreased by 1 , we obtain $H_{k+1}(-3(k+1)-16+4 i), 2 \leq i \leq \frac{3(k+1)+13}{2}$.

(3). In $H_{k}(3 k+18)$, embed $\left[\begin{array}{l}1 \\ 0\end{array}\right]+\left[\begin{array}{ll}0 & 1 \\ 1 & 0\end{array}\right]$, then $e(1)-e(0)$ decreased by 1 , we obtain $H_{k+1}(3(k+1)+14)$.

(4). In $H_{k}(3 k+14)$, embed $\left[\begin{array}{l}0 \\ 1\end{array}\right]+\left[\begin{array}{ll}1 & 1 \\ 0 & 0\end{array}\right]$, then $e(1)-e(0)$ increased by 7 , we obtain $H_{k+1}(3(k+1)+18)$.

As mentioned above, we have $F F I\left(H_{k+1}\right)=\left\{-3(k+1)-16+4 i: 1 \leq i \leq \frac{3(k+1)+17}{2}\right\}$, which complete the proof of Lemma 9.

Combining Lemma 8 and Lemma 9, 
Theorem 10. The full friendly index sets of $H_{m}$ is

$$
F F I\left(H_{m}\right)= \begin{cases}-3 m-18+4 i: & 1 \leq i \leq \frac{3 m+18}{2} m \quad \text { is even } \\ -3 m-16+4 i: & 1 \leq i \leq \frac{3 m+17}{2} m \quad \text { is odd }\end{cases}
$$

In [7], Hovey introduced the notion of $A$-cordial labelings, which generalized the concept of cordial graphs of Cahit [7]. A graph $G$ is cordial if the number of edges labeled 0 and the number of edges labeled 1 differs by at most by 1 under some friendly labeling $f$.

By Theorem 10, we can derive the following corollary.

Corollary 11. $H_{m}$ is cordial iff $m \neq 0 \quad(\bmod 4)$.

\section{Conclusion}

We obtained the full friendly index sets of a family of cubic graph graphs by subgraph embedding method. In the future, we hope get more results about cubic graphs and use this method solve other problems of graph labeling.

\section{References}

[1] I. Cahit, Cordial graphs: a weaker version of graceful and harmonious graphs, Ars Combin., 23 (1987) 201-207.

[2] Z. B. Gao, R. Y. Han, S. M. Lee, H. N. Reb, G. C. Lau, A new approach in finding full friendly indices, Bull. Malays. Math. Sci. Soc., 41 (2018) 443-453.

[3] Z. B. Gao, R. Y. Han, S. M. Lee, H. N. Reb, G. C. Lau, Labeling subgraph embeddings and cordiality of Graphs. Iranian Journal of Mathematical Sciences and Informatics., 2 (2019) 79-92.

[4] Z. B. Gao, G. Y. Sun, S. M. Lee, On full friendly index sets of 1-level and 2-levels N-grids, Discrete Appl. Math., 211 (2016) $68-78$.

[5] J. F. Hou, H. W. Ma, X. X. Yu and X. Zhang, A bound on judicious of directed graphs, Sci. China Math, 63 (2020) $297-308$.

[6] J. F. Hou, and Q. H. Zeng, On a problem of judicious k-Partitions of graphs. J. Graph Theory 85 (2017) 619-643.

[7] M. Hovey, A-cordial graphs, Discrete Math., 93 (1991) 183-194.

[8] H. F. Law, Full friendly index sets of spiders, Ars Combin., 119 (2015) 23-31.

[9] G. Y. Sun, Z. B. Gao, and S. M. Lee, On full friendly index sets of twisted product of M* obius ladders, Ars Combin., 128 (2016) 225-239.

[10] W. C. Shiu and M.-H. Ho, Full friendly index sets of slender and flat cylinder graphs, Transactions Combin., 2 (4) (2013) $63-$ 80.

[11] E. Salehi and S. M. Lee, On friendly index sets of trees, Cong. Numer., 178 (2006) 173-183.

[12] W. C. Shiu and S. M. Lee, Full friendly index sets and full product-cordial index sets of twisted cylinders., J. Combin. Number Thoery, 3 (3) (2012) 209-216.

[13] W. C. Shiu and M. H. Ling, Full friendly index sets of Cartesian products of two cycles, Acta Mathematica Sinica, English Series, 26 (2010) 1233-1244.

[14] D. Sinha and J. Kaur, Full friendly index set-I, Discrete Appl. Math., 161(9) (2013) 1262-1274.

[15] D. Sinha and J. Kaur, Full friendly index set-II, J. Combin. Math. Combin. Comput., 79 (2011) 65-75.

[16] W. C. Shiu and H. Kwong, Full friendly index sets of P2× Pn, Discrete Math.,308 (2008) 3688-3693.

[17] W. C. Shiu and F. S. Wong, Full friendly index sets of cylinder graphs, Aus-tralasian J. Combin., 52 (2012) 141-162.

[18] Y. R. Ji and J. M. Liu, On computing the edge-balanced index sets of the circle union graph F (3, n), International Journal of Modern Education and Computer Science (IJMECS)., 8(3) (2016) 22-27.

[19] Y. J. Qin and Y. G. Zheng, On The Edge-balance Index Sets of the Power Circle Nested Graph $C_{-}\left(7^{\wedge} \mathrm{m}\right) \times \mathrm{P}_{-}\left(\mathrm{m} \_7\right)(\mathrm{m} \equiv$ 2(mod3)), International Journal of Intelligent Systems and Applications(IJISA)., 6(7) (2014) 22-28.

\section{Authors' Profiles}

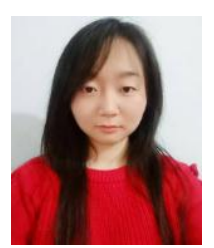

Yujie. Bai is now pursuing her master degree in the School of Mathematics and Information Science at Henan Polytechnic University, China. Her research interests include enumeration in graph theory. 
Shufei. Wu received the $\mathrm{PhD}$ degree from Fuzhou University, China in 2017. He is currently a lecture in the School of Mathematics and Information Science at Henan Polytechnic University, China. His research interests include directed graphs, hyper-graphs and probabilistic methods in extremal combinatorics.

How to cite this paper: Yujie. Bai, Shufei. Wu," Labeling a kind of Cubic Graphs by Subgraph Embedding Method ", International Journal of Mathematical Sciences and Computing(IJMSC), Vol.7, No.1, pp.1-10, 2021. DOI: 10.5815/ijmsc.2021.01.01 